\title{
A global historical ozone data set and signatures of El Niño and the 11-yr solar cycle
}

Stefan Brönnimann, Jonas Bhend, Jörg Franke, Simon Flückiger, Andreas Fischer, René Bleisch, Greg Bodeker, Birgit Hassler, Eugene Rozanov, Martin Schraner

\section{Supplementary material}

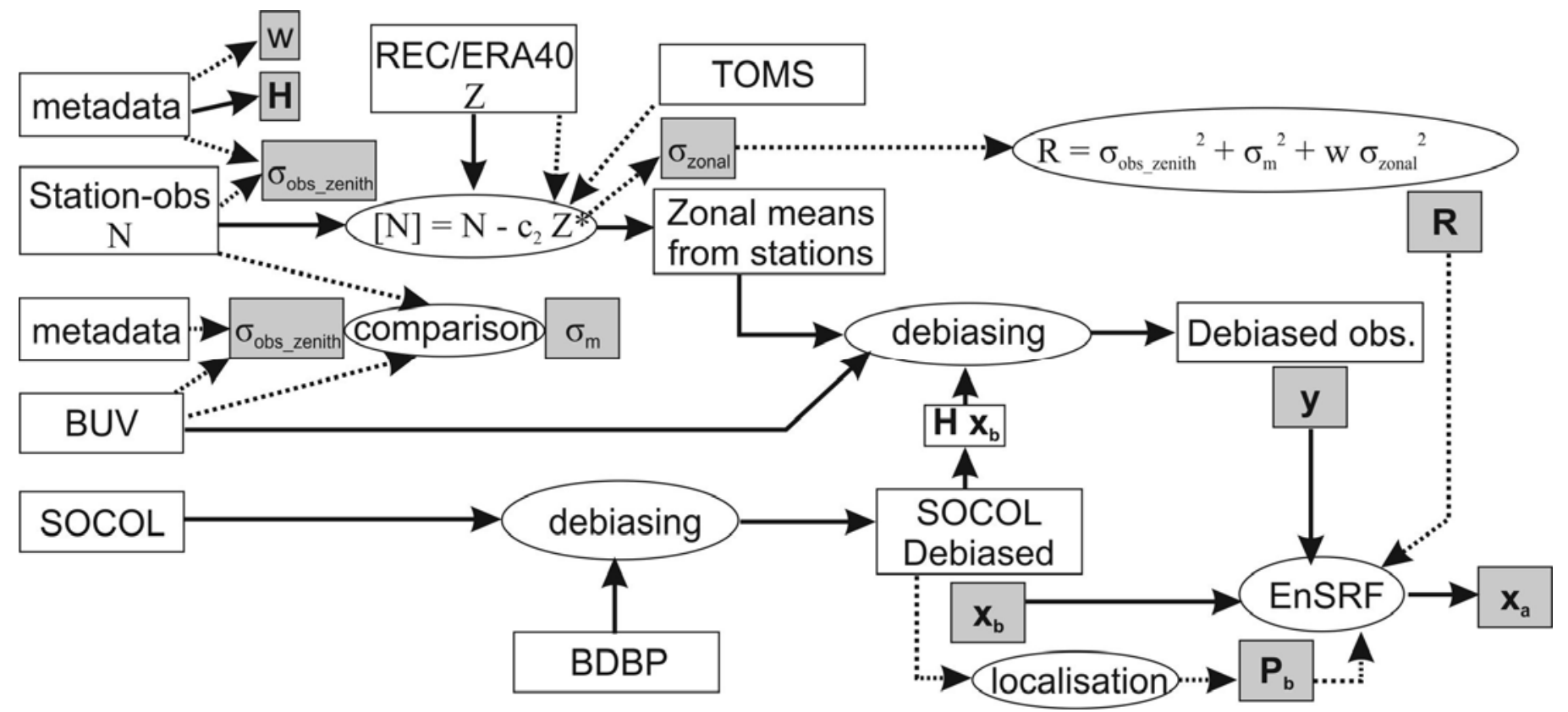

Figure S1. Schematic depiction of the approach. 

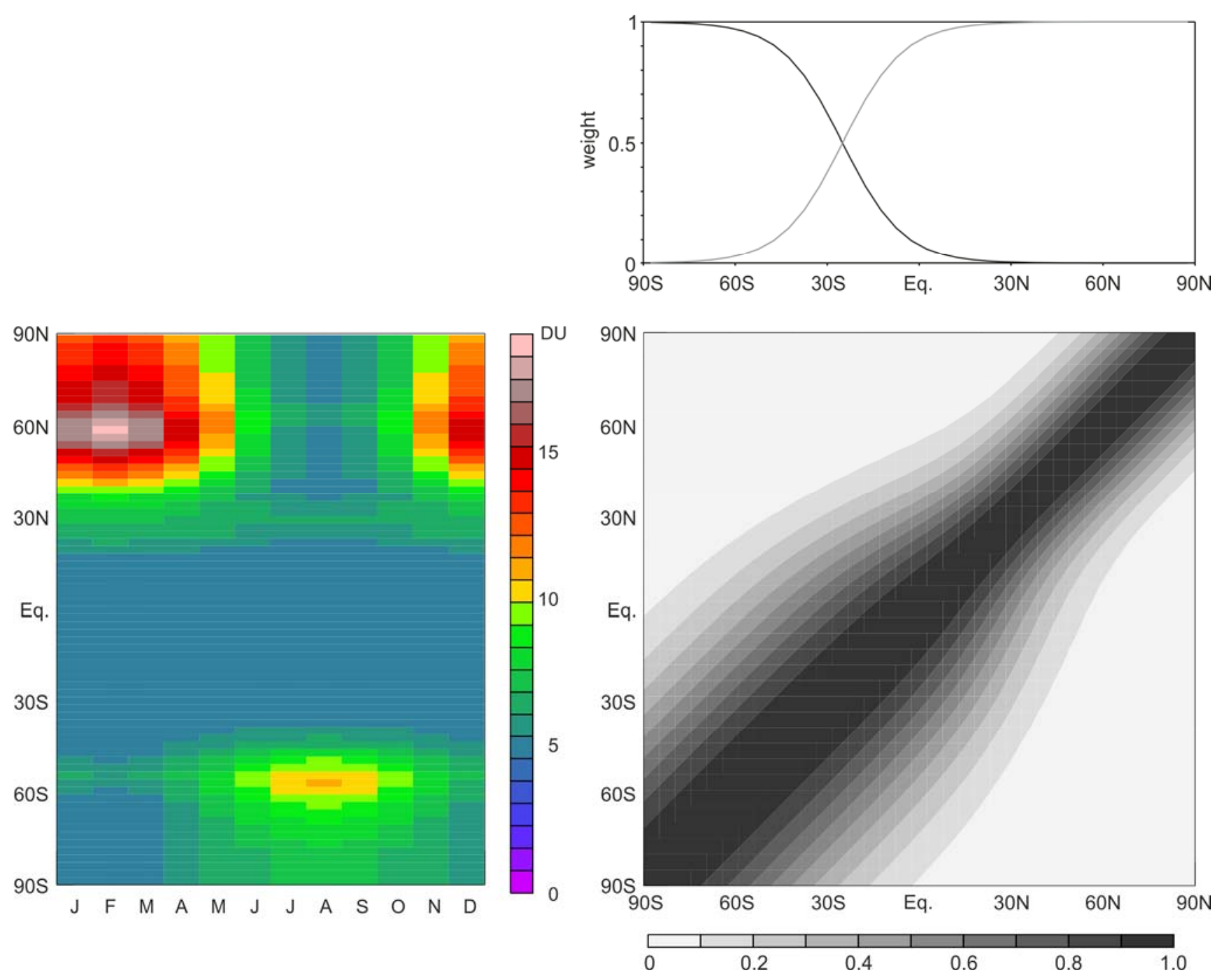

Figure S2. Left: Error of the zonal adjustment as a function of calendar month and latitude. Right: Localisation weights of the background error covariance matrix. The top panel shows the function $a$ (grey is for boreal winter, black is for aural winter) used to determine the weights. 

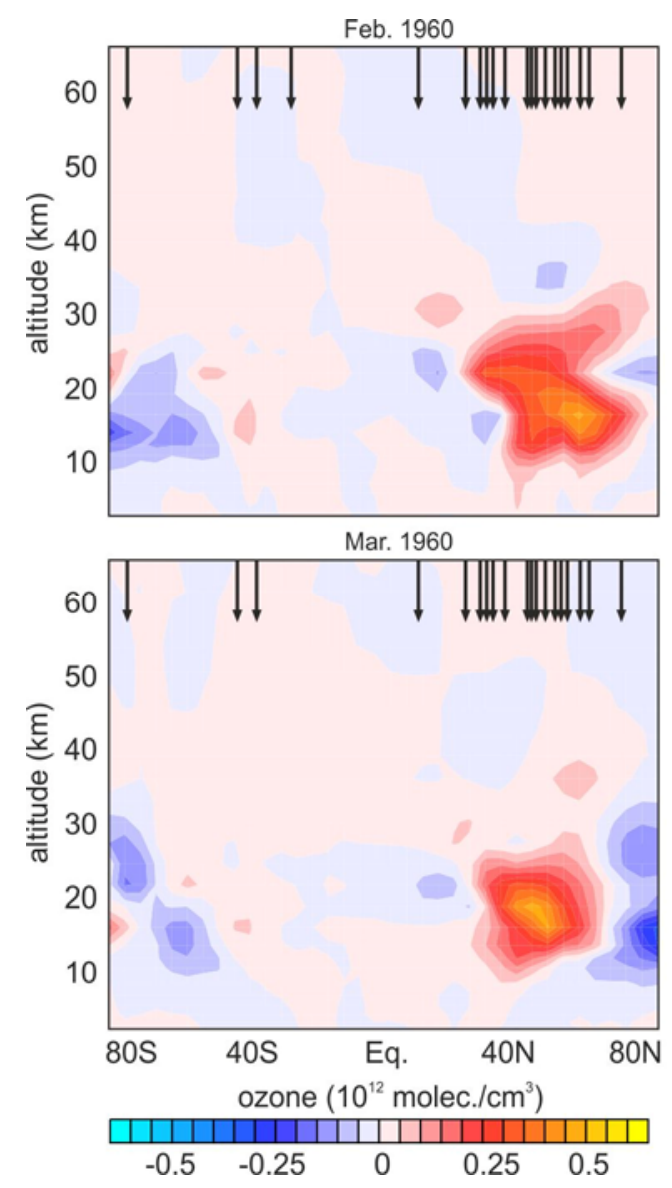
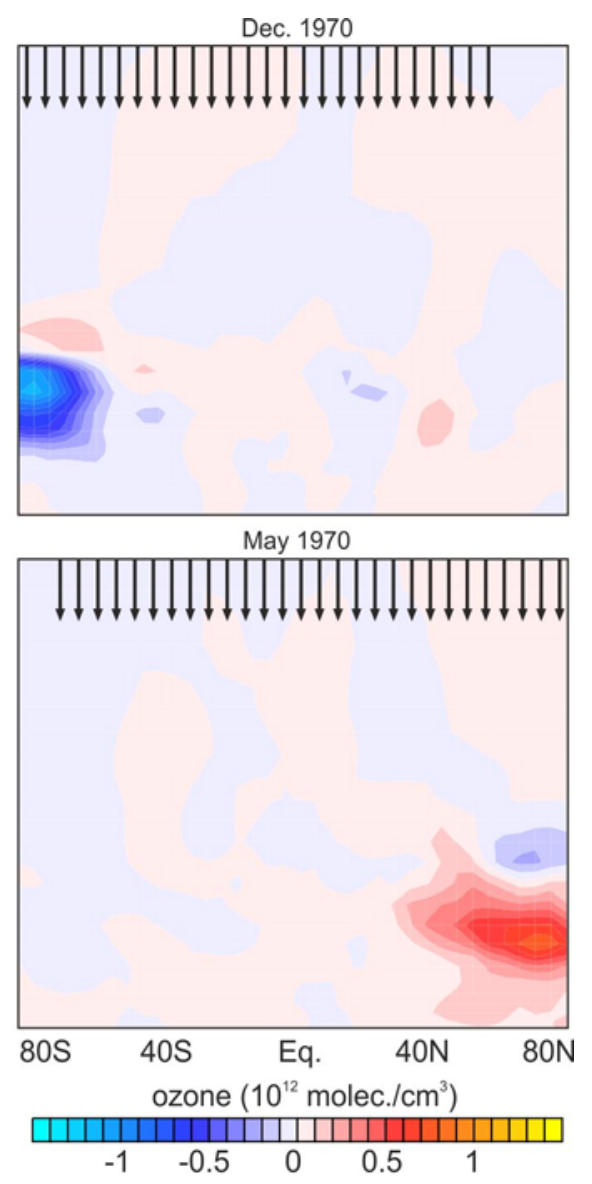

Figure S3. Results of the assimilation. Shown are the differences (assimilation minus background) for four sample months. Arrows indicate locations where observations (groundbased in the left two cases, BUV in the right two cases, note the different scale) were assimilated. 

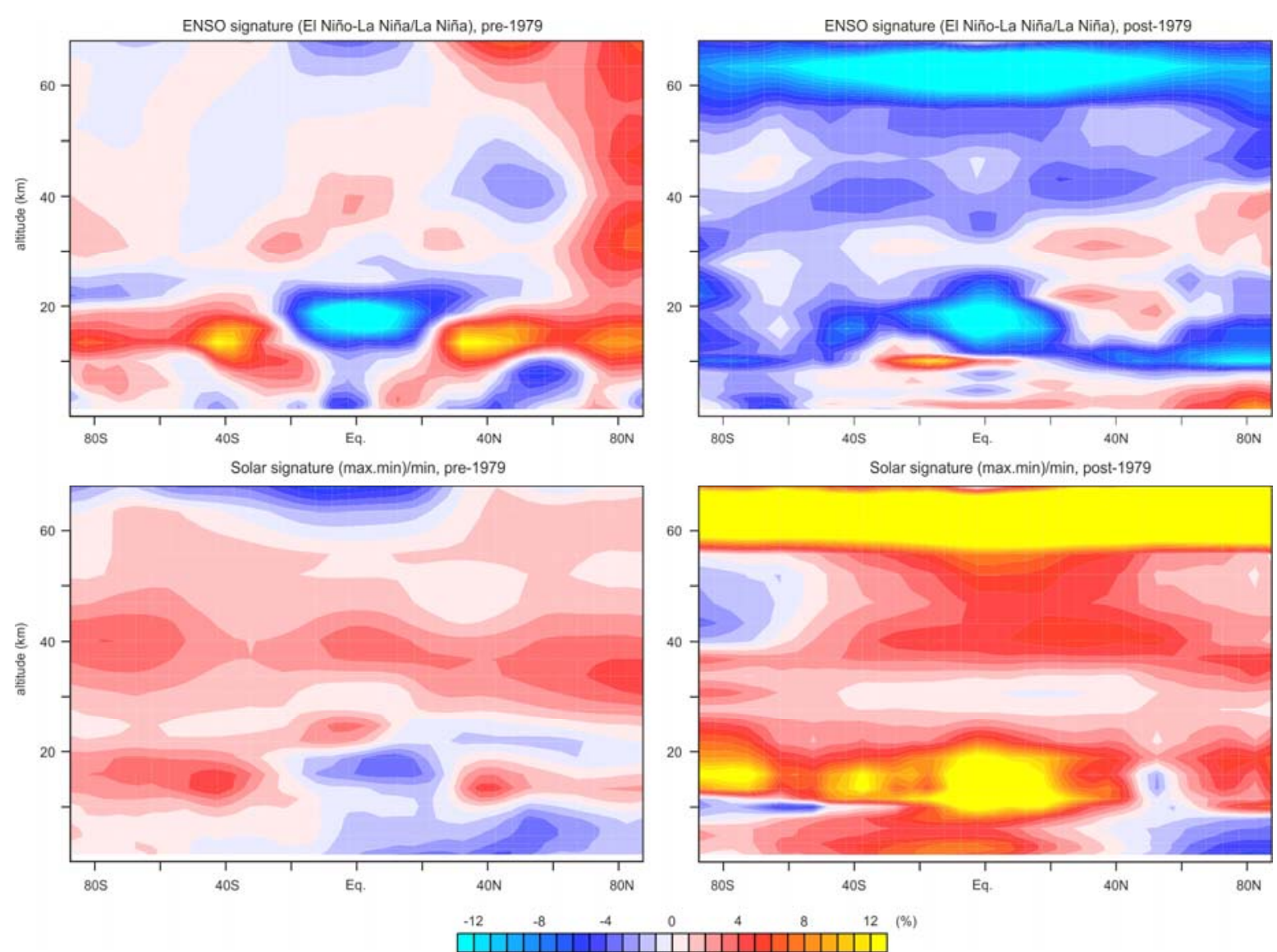

Figure S4. (top) Differences in ozone between El Niño and La Niña events in the pre-1979 period in HISTOZ (left) and the post-1979 period in BDBP. (bottom) Differences in ozone between solar maxima and solar minima in the pre-1979 period in HISTOZ (left) and the post-1979 period in BDBP. Note that the figures are the same as in Fig. 10 and 11, but plotted in terms of relative rather than absolute differences. 
Table S1. List of stations with ground-based total ozone observatios used for the assimilation. Station start years in bracketrs denote changes in the instrument. Error 1 and 2 refer to the pre-1957 and post-1957 periods.

\begin{tabular}{|c|c|c|c|c|c|c|c|c|}
\hline Station name & $\operatorname{lon}\left({ }^{\circ} \mathrm{E}\right)$ & lat $\left({ }^{\circ} \mathrm{N}\right)$ & Start Year & $\mathrm{n}$ & Reference (pre-IGY only) & Source & error1 & error2 \\
\hline Aarhus & 10.6 & 56.3 & $1940(1952)$ & 227 & Brönnimann et al. 2003ab & WOUDC & 16 & 6 \\
\hline Aldergrove & \begin{tabular}{|c|}
-6.2 \\
\end{tabular} & 54.7 & 1952 & 54 & Brönnimann et al. 2003ab & WOUDC & 4 & 6 \\
\hline Alma-Ata & 76.9 & 43.3 & 1974 & 58 & & WOUDC & 8 & 6 \\
\hline Arosa & 9.7 & 46.8 & 1926 & 588 & Staehelin et al. 1998 & WOUDC & 4 & 4 \\
\hline Aspendale & 145.1 & -38.0 & 1957 & 256 & & WOUDC & 8 & 6 \\
\hline Belsk & 20.8 & 51.8 & 1963 & 185 & & WOUDC & 8 & 6 \\
\hline Bismarck & -46.8 & 46.8 & 1957 & 216 & & WOUDC & 8 & 6 \\
\hline Bombay & 72.9 & 18.9 & 1936 & 17 & Brönnimann et al. 2003ab & WOUDC & 12 & 6 \\
\hline Brisbane & 153.1 & -27.4 & 1957 & 254 & & WOUDC & 8 & 6 \\
\hline Buenos Aires & -58.5 & -34.6 & 1965 & 156 & & WOUDC & 8 & 6 \\
\hline Cairo & 31.3 & 30.1 & 1928 & 135 & Brönnimann et al. 2003ab & internal & 8 & 6 \\
\hline Camborne & -5.3 & 50.2 & 1952 & 176 & Brönnimann et al. 2003ab & WOUDC & 12 & 6 \\
\hline Canberra & 149.0 & -35.3 & 1929 & 45 & Brönnimann et al. 2003ab & internal & 12 & 6 \\
\hline Caribou & \begin{tabular}{|c|}
-68.1 \\
\end{tabular} & 46.9 & 1958 & 199 & & WOUDC & 8 & 6 \\
\hline Christchurch & 172.6 & -43.5 & 1928 & 116 & Brönnimann et al. 2003ab & WOUDC & 8 & 6 \\
\hline College & -147.5 & 64.7 & 1952 & 78 & Brönnimann et al. 2003ab & WOUDC & 16 & 6 \\
\hline Darwin & 130.9 & -12.4 & 1966 & 100 & & WOUDC & 8 & 6 \\
\hline Dombas & 9.1 & 62.1 & 1940 & 67 & Svendby et al. 2003 & WOUDC & 8 & 6 \\
\hline Edmonton & -113.5 & 53.6 & 1950 & 282 & & WOUDC & 8 & 6 \\
\hline Flagstaff & -111.7 & 35.2 & 1954 & 82 & & internal & 8 & 6 \\
\hline HalleyBay & -26.7 & -75.5 & 1957 & 94 & & WOUDC & 8 & 6 \\
\hline Hemsby & 1.7 & 52.7 & 1952 & 36 & Brönnimann et al. 2003ab & WOUDC & 8 & 6 \\
\hline Hobart* & 147.5 & -42.8 & 1967 (1973) & 120 & & WOUDC & 8 & 6 \\
\hline Huancayo & -75.3 & -12.0 & 1964 & 176 & & WOUDC & 8 & 6 \\
\hline Kagoshima & 130.5 & 31.7 & 1958 & 236 & & WOUDC & 8 & 6 \\
\hline King Edward Point & -36.5 & -54.2 & 1971 & 91 & & WOUDC & 8 & 6 \\
\hline Kodaikanal & 77.5 & 10.2 & 1928 & 245 & Brönnimann et al. 2003ab & internal & 8 & 6 \\
\hline Lerwick & -1.2 & 60.1 & $1926(1952)$ & 295 & Brönnimann et al. 2003ab & internal & 4 & 6 \\
\hline Magny & 2.1 & 48.7 & 1955 & 75 & Brönnimann et al. 2003ab & WOUDC & 8 & 6 \\
\hline Marseille & 5.4 & 43.3 & 1927 & 22 & Brönnimann et al. 2003ab & internal & 8 & 6 \\
\hline Mauna Loa & -155.6 & 19.5 & 8 & 203 & & WOUDC & 8 & 6 \\
\hline Minamitorishima & 153.9 & 24.3 & 1958 & 66 & & WOUDC & 8 & 6 \\
\hline Mount Abu & 72.7 & 24.6 & 1951 & 197 & Brönnimann et al. 2003ab & WOUDC & 12 & 6 \\
\hline Nashville & -86.6 & 36.3 & 1962 & 188 & & WOUDC & 8 & 6 \\
\hline New Delhi & 77.2 & 28.6 & 1955 & 284 & & WOUDC & 12 & 6 \\
\hline New York & -73.9 & 40.9 & 1941 & 42 & Brönnimann et al. 2003ab & internal & 16 & 6 \\
\hline Olso & 10.7 & 60.0 & 1946 & 55 & Svendby et al. 2003 & WOUDC & 8 & 6 \\
\hline Oxford & -1.2 & 51.8 & 1925 & 403 & Vogler et al. 2006 & WOUDC & 4 & 4 \\
\hline Perth & 115.9 & -31.9 & 1969 & 113 & & WOUDC & 8 & 6 \\
\hline Potsdam & 13.0 & 52.3 & 1926 (1957) & 175 & & WOUDC & 8 & 6 \\
\hline Quetta & 66.5 & 30.1 & 1958 & 170 & & WOUDC & 8 & 6 \\
\hline Rome & 12.2 & 42.1 & 1954 & 285 & Brönnimann et al. 2003ab & WOUDC & 8 & 6 \\
\hline Sapporo & 141.3 & 43.1 & 1958 & 249 & & WOUDC & 8 & 6 \\
\hline Spitsbergen & 15.0 & 78.0 & 1950 & 127 & Vogler et al. 2005 & WOUDC & 8 & 6 \\
\hline Sterling & -77.5 & 39.0 & 1957 & 84 & & WOUDC & 8 & 6 \\
\hline Table Mountain & -117.3 & 34.1 & 1926 & 14 & Brönnimann 2005 & internal & 20 & 6 \\
\hline Table Mountain & -117.3 & 34.1 & 1928 & 13 & & internal & 8 & 6 \\
\hline Taipeh & 121.4 & 25.1 & 1965 & 76 & & WOUDC & 8 & 6 \\
\hline Tallahassee & -84.4 & 30.4 & 1964 & 134 & & WOUDC & 8 & 6 \\
\hline Tateno & 140.1 & 36.1 & 1955 & 277 & Brönnimann et al. 2003ab & internal/WOUDC & 8 & 6 \\
\hline Toronto & -79.5 & 43.8 & 1960 & 220 & & WOUDC & 8 & 6 \\
\hline Tromsoe & 19.0 & 69.7 & 1935 & 344 & Hansen and Svenoe 2005 & WOUDC & 4 & 4 \\
\hline Uppsala & 17.6 & 59.9 & 1951 & 175 & & WOUDC & 8 & 6 \\
\hline Varanasi & 83.0 & 25.3 & 1957 & 170 & & WOUDC & 8 & 6 \\
\hline Vladivostok & 131.9 & 43.1 & 1973 & 66 & & WOUDC & 8 & 6 \\
\hline ZiKaWei & 121.4 & 31.2 & 1932 & 122 & Brönnimann et al. 2003ab & WOUDC & 16 & 6 \\
\hline
\end{tabular}


